\title{
Performance Analysis of Non-uniform Sparse Segmentation Integral Method Based on Gauss Integral in EM Forward of Electrical Antenna Under Stratified Ocean
}

\author{
Zongyang Shi ${ }^{1,}$, Yiyu Zhao ${ }^{1}$, Xiaodong $\mathrm{Qu}^{2}$, Lichao Ma ${ }^{1}$ \\ ${ }^{1}$ Beijing Institute of Mechanical Equipment, Beijing, China \\ ${ }^{2}$ Aerospace Information Research Institute, Chinese Academy of Sciences, Beijing, China
}

Email address:

shizongyang13@mails.ucas.ac.cn (Zongyang Shi), zhyznd@163.com (Yiyu Zhao),xdqu@mail.ie.ac.cn (Xiaodong Qu)

${ }^{*}$ Corresponding author

To cite this article:

Zongyang Shi, Yiyu Zhao, Xiaodong Qu, Lichao Ma. Performance Analysis of Non-uniform Sparse Segmentation Integral Method Based on Gauss Integral in EM Forward of Electrical Antenna Under Stratified Ocean. Science Discovery. Vol. 9, No. 2, 2021, pp. 47-57.

doi: $10.11648 /$ j.sd.20210902.16

Received: February 18, 2021; Accepted: April 15, 2021; Published: April 26, 2021

\begin{abstract}
In order to solve the problem that it is difficult to take into account the computational efficiency and accuracy of electromagnetic field froward modeling of electrical antenna under the stratified ocean, evaluate the performance of two non-uniform sparse segmentation integral calculation methods based on Gauss integrals and improve the efficiency and accuracy of data interpretation in engineering applications, firstly two non-uniform sparse segmentation calculation methods based on Gauss integral nodes (Gauss-Legendre integral nodes and Gauss-Chebyshev integral nodes) are introduced. Then, two fast numerical integration methods corresponding of the time and frequency domain of the electrical antenna electromagnetic field under stratified ocean is established. Finally, by comparing and analyzing the results of the traditional uniform dense segmentation numerical integral calculation from the two aspects of computational accuracy and efficiency, the results show that two non-uniform sparse segmentation integral methods can improve the computational efficiency under the condition that the calculation accuracy is comparable, and the non-uniform sparse integral calculation method based on the Gauss-Chebyshev integral nodes is more significant than the calculation efficiency performance of the method based on the Gauss-Legendre integral node. At the same time, the general parameter setting of the non-uniform sparse segmentation integral method is given in this paper.
\end{abstract}

Keywords: Electric Antenna Under Water, Electromagnetic Forward of Electric Antenna,

Non-uniform Sparse Segmentation Integration Method, Gauss-Legendre, Gauss-Chebyshev

\section{基于高斯积分的非均匀稀疏分割积分方法在层状海洋模型电性天 线电磁场正演计算中的性能分析}

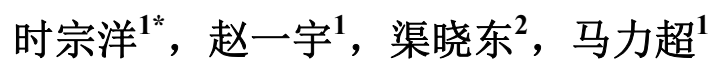

${ }^{1}$ 北京机械设备研究所, 北京, 中国

${ }^{2}$ 中国科学院空天信息创新研究院, 北京, 中国

邮箱

shizongyang13@mails.ucas.ac.cn（时宗洋），zhyznd@163.com（赵一宇），xdqu@mail.ie.ac.cn（渠晓东） 
摘要：为解决层状海洋模型下电性天线辐射电磁场正演计算效率和精度难以兼顾的问题，评估两种基于高斯积分节点 的非均匀稀疏分割积分计算方法的性能，提升工程应用中数据解释的效率和准确性，论文首先引入两种基于高斯积分 节点（高斯切比雪夫积分节点及高斯勒让德积分节点）的非均匀稀疏分割计算方法, 然后建立了对应的层状海洋电性 天线电磁场时频域正演计算的快速数值积分方法, 最后通过从计算精度和效率两个角度与传统均匀密集分割数值积分 计算结果进行对比分析。结果表明, 在计算精度相当的前提下, 两种非均匀稀疏分割积分方法均能提升计算效率, 且 基于高斯切比雪夫积分节点的非均匀稀疏积分方法较之与基于高斯勒让德积分节点方法的计算效率性能提升更显著。 论文同时给出了非均匀稀疏分割积分方法的一般参数设置。

关键词: 水下电性天线, 电性天线电磁正演计算, 非均匀稀疏分割, 高斯-勒让德, 高斯-切比雪夫

\section{1. 引言}

典型层状海洋模型下电性天线电磁场正演计算方法 主要应用海洋目标电磁探测应用领域, 如海底石油、水合 物等探测和海洋大型目标如沉船等探测等。

以海洋可控源电磁方法 (Marine Controlled Source Electromagnetic Method, MCSEM）为例, 其通常采用几百 米长的水平电性天线在海水中 (距海底几十米的位置) 辐 射峰值电流几百安培至千安培、基频在 $n \times 10^{-1} \mathrm{~Hz}$ 至 $n \times 10$ $\mathrm{Hz}$ 范围内的矩形波电流, 通过布置在海底或者拖曳在距天 线固定偏移距的电场或磁场传感器观测电场/磁场响应信 号[1-2], 然后通过采用相应数据处理手段对电磁信号进行 处理, 运用层状海洋模型正反演算法获得对实测电磁信号 的定量反演解释, 从而得到海洋中目标电阻率信息, 图1 给出了海洋可控源电磁方法示意图, 图中示意的传感器阵 列位于海底沿电性天线轴向布置, 实际应用中传感器根据 需要可以布置于海水中或海水表面。

当前实际工程应用中, 通常基于1D层状海洋模型电性 天线电磁场正演计算方法进行空间中（海水、空气）的电 磁计算, 以实现勘探区域海水及海底的电导率反演计算。 其中传统的1D层状海洋电性天线电磁场正演计算方法主 要有两种, 一种是将有限长电性天线看作电偶极子天线 [3-5, 6, 8], 利用层状海洋模型电偶极天线电磁场模拟解释 海洋目标及海底电性参数; 另一种方法是将有限长度电性 天线进行均匀密集分割[9], 将分割后每一段等效为电偶极 子, 再对每个等效电偶极子天线的电磁响应求和得到有限 长电性天线的辐射电磁场。

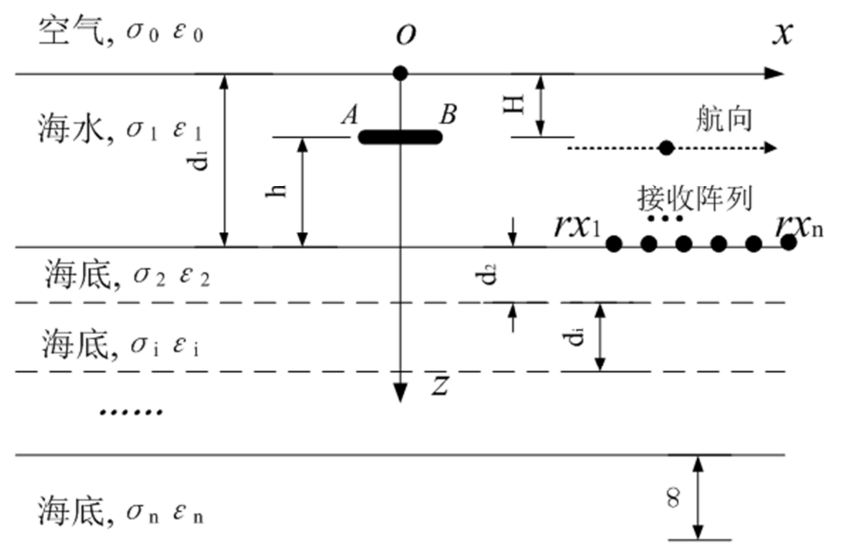

图 1 海洋可控源电磁方法 (MCSEM) 示意图。
传统的层状海洋模型电性天线电磁场正演计算方法 存在如下不足之处, 方法一将有限长电性天线看作电偶极 子天线, 这种方法认为, 当海洋目标（海底或海水目标） 和观测传感器距离电性天线很远 (相比于电性天线长度) 时电性天线可以等效为电偶极子天线。然而, 实际MCSEM 中的电性天线长几百米 (一般100米至300米), 距离海底 几十米距离, 航行作业时, 电性天线从靠近海底的传感器 阵列到远离传感器阵列的过程中, 无法始终保证电性天线 距离海底和传感器的距离远大于电性天线的长度（如5倍 电性天线长度), 从而导致该方法在计算小偏移距的电磁 场时产生较大的计算误差。方法二均匀密集分割有限长度 电性天线, 然后把分割后的每段等效为电偶极子, 并对每 个等效电偶极子天线的电磁响应求和得到有限长电性天 线的辐射电磁场。该方法在保证计算精度的同时会极大的 降低计算效率, 不利于工程应用中数据资料的快速解释 [10]。

针对传统的层状海洋电性天线电磁场正演计算难以 兼顾计算精度和计算效率的问题, 本文提出了两种基于高 斯积分节点的非均匀稀疏分割计算方法实现层状海洋电 性天线电磁场计算效率的提升。两种非均匀稀疏分割积分 方法分别采用勒让德积多项式零点和切比雪夫多项式的 零点作为非均匀分割分点进行离散化, 然后进行数值积分 计算。这两种非均匀稀疏分割计算方法均能够实现限长电 性天线电磁场的精确快速计算, 兼顾了计算的精度和效率。 通过对比分析两种方法的计算精度和效率, 发现基于高斯 -切比雪夫积分节点的非均匀稀疏分割计算方法的计算效 率较之于基于高斯-勒让德积分节点的计算方法有显著效 率提升。

论文首先介绍层状海洋模型电性天线电磁场的工程 应用及传统计算方法及其存在的不足, 引出本论文建立的 非均匀稀疏分割积分方法及其优势; 第二部分构建了层状 海洋模型电磁天线辐射电磁场模型, 并对电磁场积分计算 式进行了理论推导, 然后分别建立基于高斯-勒让德分点 和高斯-切比雪夫分点的非均匀稀疏分割积分方法, 给出 理论计算式和算法流程; 第三部分通过模拟仿真算例, 对 电偶极子等效计算方法、密集均匀分割积分方法和本论文 提出的两种非均匀稀疏分割数值积分方法这四种方法在 电磁场计算精度和效率方面进行对比分析, 论证了本论文 提出方法在兼顾计算精度和效率方面的优势, 基于高斯切 比雪夫分点的非均匀稀疏分割数值积分方法能够实现工 程应用中数据解释的效率和准确性的提升; 第四部分对全 文进行总结, 给出论文结论。 


\section{2. 层状海洋模型电性天线辐射电磁场理论}

\section{1. 典型层状海洋模型}

以三层海洋模型 (空气层、海水层和海底层) 为例, 其中空气层设定为半无限大均匀空间, 海底层可以为半无 限大均匀空间或层状空间, 海水层深度为 $d_{1}$, 设定分解面 互相平行, 水平方向上无限延伸, 空气、海水和海底（可 以为多层海底) 的介电参数分别为 $\sigma_{i} 、 \varepsilon_{i}$ $(i=0,1,2, \cdots, n, n \geq 2)$ ，其中 $i=0$ 表示空气层， $i=1$ 表 示海水层。真空磁导率为 $\mu_{0}$, 相对磁导率 $\mu_{r}$ 设置为 1 。 $\mathrm{AB}$ 表示电性天线首尾, 天线长度为 $L$, 天线电极矩为 $P=I L$, 天线中点位于原点 $O$ 正下方, 目标所在深度为 $H$, 距离海底深度为 $h$, 各层厚度为 $d_{i}$ 。笛卡尔坐标系正 $z$ 方 向垂直水平面向下, $o-x y z$ 满足右手螺旋定则。假设任意 姿态电性天线 (见图2) 在 $X O Y$ 平面内的投影与 $x$ 轴夹角 为 $\theta$, 天线与 $X O Y$ 平面的夹角为 $\phi$ 。

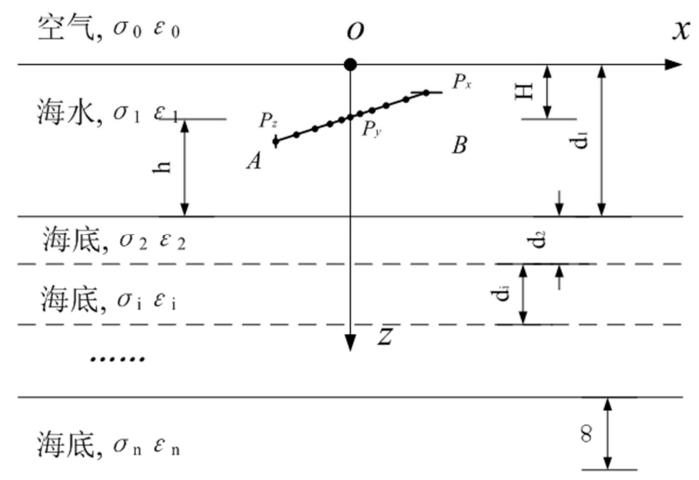

图2 层状海洋电性天线及非均匀稀疏分点示意图。

层状海洋电性天线模型见图2。层状海洋电性天线电 磁场的推导基于以下假设[11-13]:

a) 满足准静态近似条件，频率小于 $100 \mathrm{kHz}$;

b) 准静态近似下忽略海水和海底环境的位移电流, 波 数为 $k_{i}^{2}=-i \omega \mu_{0} \sigma_{i}, i=1,2, \cdots, n$ 。空气中仅存在位 移电流, 约定电导率为 $\sigma_{0}=i \omega \varepsilon_{0}$, 波数为 $k_{0}^{2}=\omega^{2} \mu_{0} \varepsilon_{0} ;$

c) 空气、海水及同层海底媒介时各向同性的, 参数与 时间、温度和压强无关;

d) 准静态近似下, 认为媒介参数与频率无关, 磁导率 和介电常数采用真空中的参数。

对于没有自由电荷的空间，电性天线在空间中产生的 电磁场满足如下麦克斯韦方程组。

$$
\left\{\begin{array}{l}
\nabla \times \mathrm{E}=j \mu \omega \mathrm{H} \\
\nabla \times \mathrm{H}=(\sigma-j \varepsilon \omega) \mathrm{E} \\
\nabla \cdot \mathrm{H}=0 \\
\nabla \cdot \mathrm{E}=0
\end{array}\right.
$$

定义电矢量为 $\mathbf{A}$, 满足 $\mathrm{H}=\nabla \times \mathrm{A}$ 。由式得到电场与电 矢位关系，

$$
\mathrm{E}=j \mu \omega \mathrm{A}-\nabla U
$$

其中 $U$ 为标量位, 满足 $U=-\frac{1}{\sigma} \nabla \cdot \mathrm{A}$ 。

\section{2. 电偶极天线辐射电磁场}

\subsection{1. 水平电偶极天线频域电磁场}

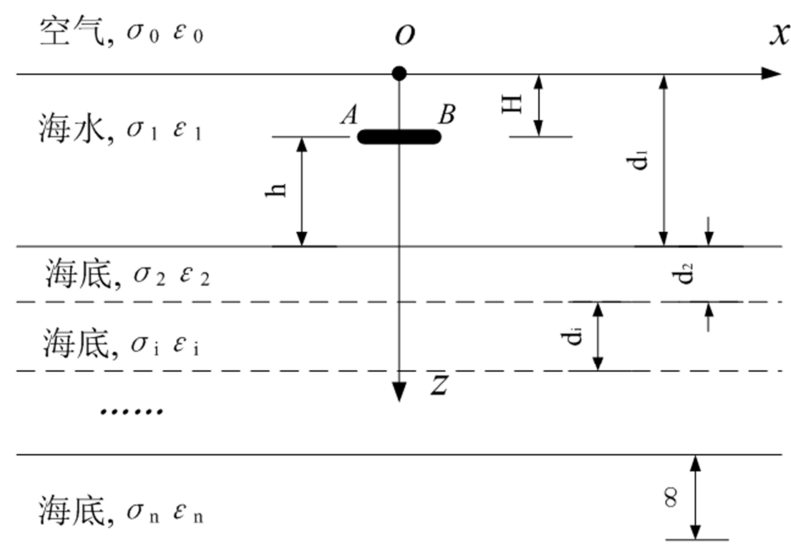

图3 层状海洋水平电偶极天线示意图。

层状海洋水平电偶极天线见图3, 其电磁场具有对称 性, 此时电矢量位 $\mathbf{A}$ 仅包含了两个分量, 即沿偶极矩方向 的分量 $A_{x}$ 和沿垂直海水-空气分解面的分量 $A_{z}$ 。电矢量位 A在笛卡尔坐标系下可表示为

$$
\mathrm{A}=\left(A_{x}, 0, A_{z}\right)
$$

矢量位、电场及磁场满足的边界条件为 [13-15]:

a) 整个空间中, 除发射源位置外, 矢量位 $\mathbf{A}$ 处处为有 限值, 且在无穷远处, 矢量位为零, 即 $\mathrm{A}(\mathrm{r} \rightarrow \infty) \rightarrow 0$;

b) 各层分解面上, 电场和磁场的切向分量连续。

根据边界条件约束, 电场 $E$ 、磁场 $H$ 与矢量为 $A$ 和 标量位 $U$ 之间的关系, 采用分离变量法可得到各层中矢量 位各分量的解。

空气中电场各分量频域计算式为:

$\left\{\begin{array}{l}E_{x 0}=\frac{I d l}{4 \pi \sigma_{0}} \int_{0}^{\infty}\left[k_{0}^{2} C_{0}+\left(B_{0} m_{0}-C_{0}\right) m^{2} \cos ^{2} \phi\right] e^{m_{0} z} J_{0}(m r) d m \\ +\frac{I d l}{4 \pi \sigma_{0}} \int_{0}^{\infty}\left(B_{0} m_{0}-C_{0}\right) m e^{m_{0} z} \frac{\sin ^{2} \phi-\cos ^{2} \phi}{r} J_{1}(m r) d m \\ E_{y 0}=\frac{I d l}{4 \pi \sigma_{0}} \sin \phi \cos \phi \int_{0}^{\infty}\left(B_{0} m_{0}-C_{0}\right) m e^{m_{0} z}\left[m J_{0}(m r)-\frac{2}{r} J_{1}(m r)\right] d m \\ E_{z 0}=\frac{I d l}{4 \pi \sigma_{0}} \cos \phi \int_{0}^{\infty}\left(B_{0} m^{2}-C_{0} m_{0}\right) m e^{m_{0} z} J_{1}(m r) d m\end{array}\right.$

空气中磁场各分量频域计算式为: 


$$
\left\{\begin{array}{l}
H_{x 0}=\frac{I d l}{4 \pi} \sin \phi \cos \phi \int_{0}^{\infty} B_{0} m e^{m_{0} z}\left(m J_{0}(m r)-\frac{2}{r} J_{1}(m r)\right) d m \\
H_{y 0}=\frac{I d l}{4 \pi} \int_{0}^{\infty}\left(m_{0} C_{0}-m^{2} \cos ^{2} \phi B_{0}\right) e^{m_{0} z} J_{0}(m r) d m \\
-\frac{I d l}{4 \pi} \int_{0}^{\infty} B_{0} m e^{m_{0} z} \frac{\sin ^{2} \phi-\cos ^{2} \phi}{r} J_{1}(m r) d m \\
H_{z 0}=\frac{I d l}{4 \pi} \sin \phi \int_{0}^{\infty} C_{0} m e^{m_{0} z} J_{1}(m r) d m
\end{array}\right.
$$

海水中电场各分量频域计算式为:

$$
\left\{\begin{array}{l}
E_{x 1}=\frac{I d l}{4 \pi \sigma_{1}} k_{1}^{2} \int_{0}^{\infty}\left[\frac{m}{m_{1}} e^{-m_{1}|z-H|}+C_{1} e^{m_{1} z}+D_{1} e^{-m_{1} z}\right] J_{0}(m r) d m \\
-\frac{I d l}{4 \pi \sigma_{1}} \int_{0}^{\infty} m\left[\left(\frac{m}{m_{1}} e^{-m_{1}|z-H|}+C_{1} e^{m_{1} z}+D_{1} e^{-m_{1} z}\right)-m_{1}\left(B_{1} e^{m_{1} z}-E_{1} e^{-m_{1} z}\right)\right] \\
{\left[\begin{array}{l}
\left.m \cos ^{2} \phi J_{0}(m r)+\frac{\sin ^{2} \phi-\cos ^{2} \phi}{r} J_{1}(m r)\right] d m \\
E_{y 1}=-\frac{I d l}{4 \pi \sigma_{1}} \sin \phi \cos \phi \int_{0}^{\infty} m\left[\left(\frac{m}{m_{1}} e^{-m_{1}|z-H|}+C_{1} e^{m_{1} z}+D_{1} e^{-m_{1} z}\right)-m_{1}\left(B_{1} e^{m_{1} z}-E_{1} e^{-m_{1} z}\right)\right] \\
{\left[\begin{array}{l}
\left.m J_{0}(m r)-\frac{2}{r} J_{1}(m r)\right] d m \\
E_{z 1}=\frac{I d l}{4 \pi \sigma_{1}} \cos \phi \int_{0}^{\infty}\left[\left(\operatorname{sgn}(z-H) m e^{-m_{1}|z-H|}-C_{1} m_{1} e^{m_{1} z}+D_{1} m_{1} e^{-m_{1} z}\right)+\right] m J_{1}(m r) d m
\end{array} m^{2}\left(B_{1} e^{m_{1} z}+E_{1} e^{-m_{1} z}\right)\right.}
\end{array} .\right.}
\end{array}\right.
$$

海水中磁场各分量频域计算式为:

$$
\left\{\begin{array}{l}
H_{x 1}=\frac{I d l}{4 \pi} \sin \phi \cos \phi \int_{0}^{\infty} m\left(B_{1} e^{m_{1} z}+E_{1} e^{-m_{1} z}\right)\left[m J_{0}(m r)-\frac{2}{r} J_{1}(m r)\right] d m \\
H_{y 1}=\frac{I d l}{4 \pi} \int_{0}^{\infty}\left[\begin{array}{l}
\left(\operatorname{sgn}(H-z) m e^{-m_{1}|z-H|}+C_{1} m_{1} e^{m_{1} z}-D_{1} m_{1} e^{-m_{1} z}\right)- \\
m^{2} \cos ^{2} \phi\left(B_{1} e^{m_{1} z}+E_{1} e^{-m_{1} z}\right)
\end{array}\right] J_{0}(m r) d m \\
-\frac{I d l}{4 \pi} \int_{0}^{\infty}\left(B_{1} e^{m_{1} z}+E_{1} e^{-m_{1} z}\right) m \frac{\sin ^{2} \phi-\cos ^{2} \phi}{r} J_{1}(m r) d m \\
H_{z 1}=\frac{I d l}{4 \pi} \sin \phi \int_{0}^{\infty}\left(\frac{m}{m_{1}} e^{-m_{1}|z-H|}+C_{1} e^{m_{1} z}+D_{1} e^{-m_{1} z}\right) m J_{1}(m r) d m
\end{array}\right.
$$

其中, $\phi$ 为观测点与电偶极源位置连线在XOY平面投影与 $x$ 轴的夹角, $\sin \phi=\frac{y}{r}, \cos \phi=\frac{x}{r}, I d l$ 表示电偶极源 的电矩, $m$ 为积分变量, $r=\sqrt{x^{2}+y^{2}}$ 为收发距, $m_{i}=\sqrt{m^{2}-k_{i}^{2}}, i=0,1,2 \ldots, N$ 。积分核中的 $J_{i}(m r), i=0,1$ 代表第 $\mathrm{i}$ 阶贝塞尔函数。参数 $C_{i} 、 B_{i} 、 D_{i}$ 和 $E_{i}$ 为待定系数, 通过理论推导计算结果如下: 


$$
\left\{\begin{array}{l}
C_{0}=\frac{m}{m_{1}} \frac{1-N_{01}}{e^{m_{1} H}} \frac{1-N_{21} e^{-2 m_{1} d_{1}} e^{2 m_{1} H}}{1-N_{21} N_{01} e^{-2 m_{1} d_{1}}} \\
B_{0}=B_{1}+E_{1} \\
C_{1}=\frac{m}{m_{1}} N_{21} \frac{e^{-2 m_{1} d_{1}}\left(N_{01} e^{-m_{1} H}-e^{m_{1} H}\right)}{1-N_{21} N_{01} e^{-2 m_{1} d_{1}}} \\
B_{1}=-\frac{P_{21} e^{-2 m_{1} d_{1}}}{m} \frac{e^{m_{1} H}+P_{01} e^{-m_{1} H}}{1-P_{01} P_{21} e^{-2 m_{1} d_{1}}}+\frac{m_{1}}{m^{2}} C_{1} \\
D_{1}=\frac{m}{m_{1}} N_{01} \frac{e^{-m_{1} H}-N_{21} e^{-2 m_{1} d_{1}} e^{m_{1} H}}{N_{21} N_{01} e^{-2 m_{1} d_{1}}-1} \\
E_{1}=\frac{P_{01} P_{21} e^{-2 m_{1} d_{1}}}{m} \frac{e^{m_{1} H}+P_{01} e^{-m_{1} H}}{1-P_{01} P_{21} e^{-2 m_{1} d_{1}}}+\frac{P_{01}}{m} e^{-m_{1} H}-\frac{m_{1}}{m^{2}} D_{1}
\end{array}\right.
$$

式中的 $N_{01} 、 N_{21} 、 P_{01}$ 和 $P_{21}$ 计算式如下:

$$
\left\{\begin{array}{c}
N_{01}=\frac{m_{0}-m_{1}}{m_{0}+m_{1}}, N_{21}=\frac{1-\frac{m_{1}}{m_{2}} R_{z}^{*}}{1+\frac{m_{1}}{m_{2}} R_{z}^{*}} \\
P_{01}=\frac{\sigma_{1} m_{0}-\sigma_{0} m_{1}}{\sigma_{1} m_{0}+\sigma_{0} m_{1}}, P_{21}=\frac{1-\frac{\rho_{1} m_{1}}{\rho_{2} m_{2}} Q_{2}^{*}}{1+\frac{\rho_{1} m_{1}}{\rho_{2} m_{2}} Q_{2}^{*}}
\end{array}\right.
$$

式中的 $R_{2}^{*}$ 和 $Q_{2}^{*}$ 的递推计算式为:

$$
\left\{\begin{array}{l}
R_{N}^{*}=1 \\
R_{k}^{*}=\frac{m_{k} R_{k+1}^{*}+m_{k+1} \tanh \left(m_{k} d_{k}\right)}{m_{k+1}+m_{k} R_{k+1}^{*} \tanh \left(m_{k} d_{k}\right)} \\
Q_{N}^{*}=1 \\
Q_{k}^{*}=\frac{\rho_{k} m_{k} Q_{(k+1)}^{*}+\rho_{k+1} m_{k+1} \tanh \left(m_{k} d_{k}\right)}{\rho_{k} m_{k} Q_{(k+1)}^{*} \tanh \left(m_{k} d_{k}\right)+\rho_{k+1} m_{k+1}}
\end{array}\right.
$$

\subsection{2. 垂直电偶极天线频域电磁场}

层状海洋垂直电偶极天线示意图见图4。

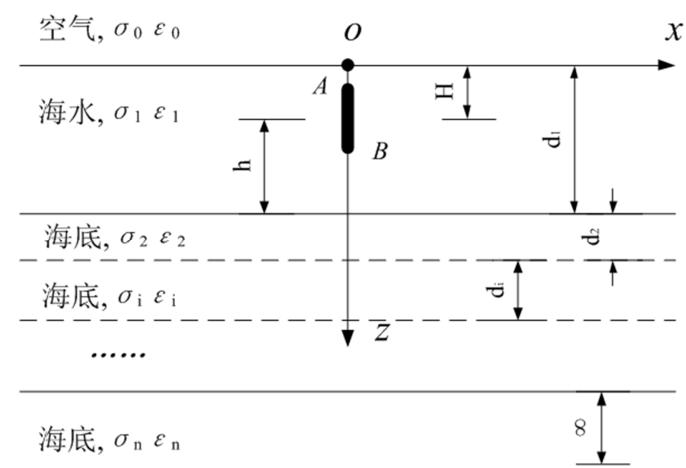

图4 层状海洋垂直电偶极天线示意图。
层状海洋垂直电偶极天线示意图见图 4, 其电矢位 $\mathbf{A}$ 仅包含沿偶极矩方向的分量 $A_{z}[16]$ 。此时的电矢量位 $\mathbf{A}$ 在 笛卡尔坐标系下表示为:

$$
\mathrm{A}=\left(0,0, A_{z}\right)
$$

根据边界条件约束, 及电场 $E$ 、磁场 $H$ 与矢量为 $A$ 和 标量位 $U$ 之间的关系, 采用分离变量法可得到各层中矢量 位各分量的解。

空气中电场各分量频域计算式为:

$$
\left\{\begin{array}{l}
E_{x 0}=-\frac{I d l}{4 \pi \sigma_{0}} \cos \phi \int_{0}^{\infty} C_{0} m m_{0} e^{m_{0} z} J_{1}(m r) d m \\
E_{y 0}=-\frac{I d l}{4 \pi \sigma_{0}} \sin \phi \int_{0}^{\infty} C_{0} m m_{0} e^{m_{0} z} J_{1}(m r) d m \\
E_{z 0}=\frac{I d l}{4 \pi \sigma_{0}} \int_{0}^{\infty} C_{0} m^{2} e^{m_{0} z} J_{0}(m r) d m
\end{array}\right.
$$

空气中磁场各分量频域计算式为:

$$
\left\{\begin{array}{l}
H_{x 0}=-\frac{I d l}{4 \pi} \sin \phi \int_{0}^{\infty} C_{0} m e^{m_{0} z} J_{1}(m r) d m \\
H_{y 0}=-\frac{I d l}{4 \pi} \cos \phi \int_{0}^{\infty} C_{0} m e^{m_{0} z} J_{1}(m r) d m
\end{array}\right.
$$

海水中电场各分量频域计算式为:

$$
\left\{\begin{array}{l}
E_{x 1}=-\frac{I d l}{4 \pi \sigma_{1}} \cos \phi \int_{0}^{\infty}\left[\begin{array}{l}
-\operatorname{sgn}(z-H) m e^{-m_{1}|z-H|}+ \\
C_{1} m_{1} e^{m_{1} z}-D_{1} m_{1} e^{-m_{1} z}
\end{array}\right] m J_{1}(m r) d m \\
E_{y 1}=-\frac{I d l}{4 \pi \sigma_{1}} \sin \phi \int_{0}^{\infty}\left[\begin{array}{l}
-\operatorname{sgn}(z-H) m e^{-m_{1}|z-H|}+ \\
C_{1} m_{1} e^{m_{1} z}-D_{1} m_{1} e^{-m_{1} z}
\end{array}\right] m J_{1}(m r) d m \\
E_{z 1}=\frac{I d l}{4 \pi \sigma_{1}} \int_{0}^{\infty}\left(\frac{m}{m_{1}} e^{-m_{1}|z-H|}+C_{1} e^{m_{1} z}+D_{1} e^{-m_{1} z}\right) m^{2} J_{0}(m r) d m
\end{array}\right.
$$

海水中磁场各分量频域计算式为: 
$\left\{\begin{array}{l}H_{x 1}=-\frac{I d l}{4 \pi} \sin \phi \int_{0}^{\infty}\left(\frac{m}{m_{1}} e^{-m_{1}|z-H|}+C_{1} e^{m_{1} z}+D_{1} e^{-m_{1} z}\right) m J_{1}(m r) d m \\ H_{y 1}=-\frac{I d l}{4 \pi} \cos \phi \int_{0}^{\infty}\left(\frac{m}{m_{1}} e^{-m_{1}|z-H|}+C_{1} e^{m_{1} z}+D_{1} e^{-m_{1} z}\right) m J_{1}(m r) d m\end{array}\right.$

其中, 参数 $C_{i}$ 和 $D_{i}$ 为待定系数, 通过理论推导计算 结果如下:

$$
\left\{\begin{array}{l}
C_{0}=\frac{m}{m_{1}} \frac{P_{01}-1}{e^{m_{1} H}} \frac{P_{21} e^{-2 m_{1} d_{1}} e^{2 m_{1} H}-1}{1-P_{21} P_{01} e^{-2 m_{1} d_{1}}} \\
C_{1}=\frac{m}{m_{1}} P_{21} \frac{e^{-2 m_{1} d_{1}}\left(P_{01} e^{-m_{1} H}-e^{m_{1} H}\right)}{1-P_{21} P_{01} e^{-2 m_{1} d_{1}}} \\
D_{1}=\frac{m}{m_{1}} P_{01} \frac{e^{-m_{1} H}-P_{21} e^{-2 m_{1} d_{1}} e^{m_{1} H}}{P_{21} P_{01} e^{-2 m_{1} d_{1}}-1}
\end{array}\right.
$$

式中的 $P_{01}$ 和 $P_{21}$ 计算式如下:

$$
P_{01}=\frac{\sigma_{1} m_{0}-\sigma_{0} m_{1}}{\sigma_{1} m_{0}+\sigma_{0} m_{1}}, P_{21}=\frac{1-\frac{\rho_{1} m_{1}}{\rho_{2} m_{2}} R_{2}^{*}}{1+\frac{\rho_{1} m_{1}}{\rho_{2} m_{2}} R_{2}^{*}}
$$

其中的 $R_{2}^{*}$ 的递推计算式如下:

$$
\left\{\begin{array}{l}
R_{N}^{*}=1 \\
R_{k}^{*}=\frac{m_{k} R_{k+1}^{*}+m_{k+1} \tanh \left(m_{k} d_{k}\right)}{m_{k+1}+m_{k} R_{k+1}^{*} \tanh \left(m_{k} d_{k}\right)}, 2 \leq k \leq N-1
\end{array}\right.
$$

\subsection{3. 任意姿态电偶极天线频域电磁场}

在计算海水中任意姿态电偶极天线辐射电磁场时, 将 偶极矩分别向 $\mathrm{x}, \mathrm{y}$ 和 $\mathrm{z}$ 轴投影, 然后分别计算三分量投影电 偶极子 $P_{x} 、 P_{y}$ 和 $P_{z}$ 的电磁场, 最后将各方向电偶极天线 的电磁场求和即可 [17-18]。层状海洋任意姿态电偶极天线 示意图见图5。

$$
\left\{\begin{array}{l}
P_{x}=P \cos \varphi \cos \theta \\
P_{y}=P \cos \varphi \sin \theta \\
P_{z}=P \sin \varphi
\end{array}\right.
$$

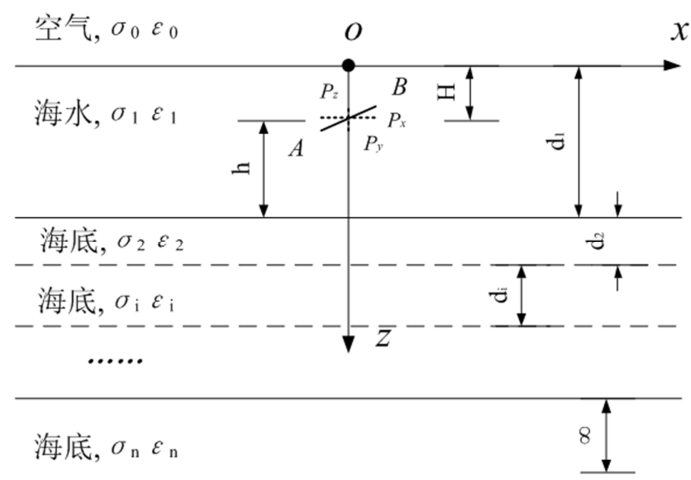

图5 层状海洋任意姿态电偶极天线示意图。
其中, 沿 $y$ 方向的电矩为 $P_{y}$ 的电偶极子天线产生的 电磁场可以通过计算沿 $x$ 方向的电矩为 $P_{y}$ 的电偶极子天 线的电磁场然后通过坐标变换得到。

\subsection{4. 电偶极天线电磁场计算方法}

层状海洋电偶极天线辐射电磁场计算方法流程见图6, 其中包含了电磁场频域和时域计算流程, 总体思路是先根 据电偶极天线辐射电磁场的频域计算式通过快速 Hankel 变换数值滤波方法实现频域计算, 获得频域电磁结果; 然 后通过GS变换数值滤波方法实现快速的频-时变换, 得到 时域电磁计算结果[19-20]。

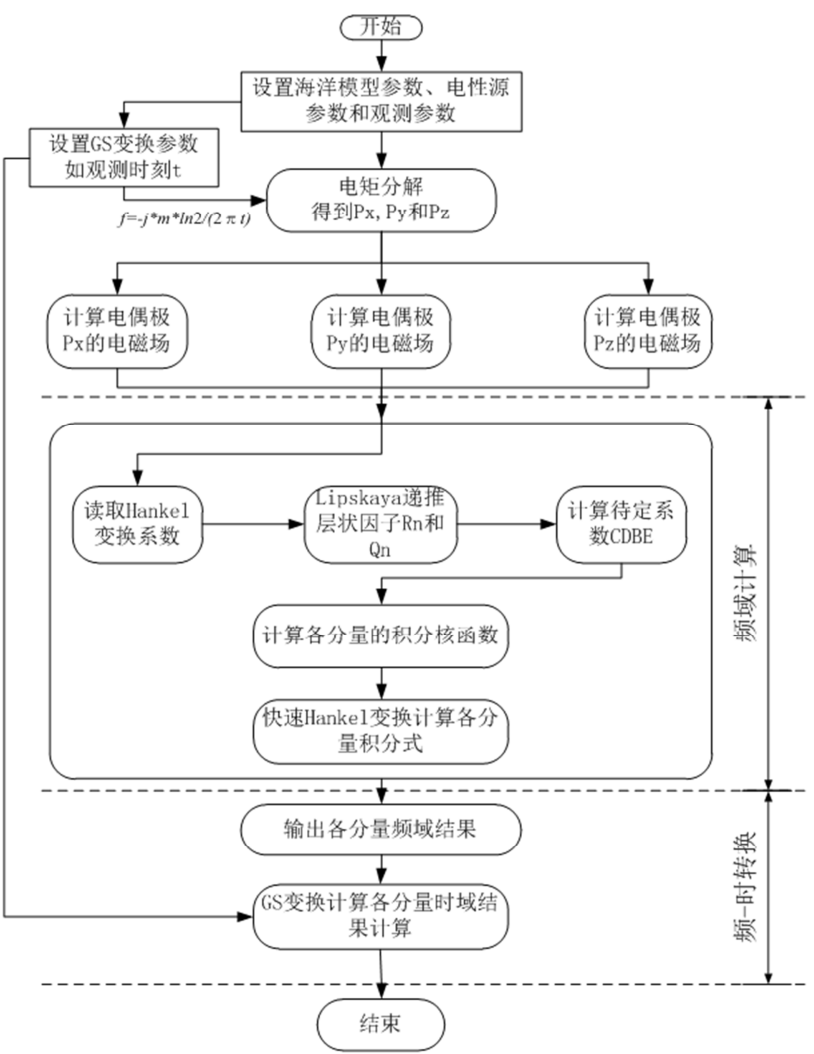

图6 层状海洋电偶极天线电磁场计算算法流程。

\section{3. 有限长度电性天线电磁场}

\subsection{1. 任意姿态电性天线电磁场的积分计算式}

理论上, 层状海洋任意姿态有限长度电性天线电磁场 的计算需要通过对天线长度进行积分获得。

假设电性天线 $l$ 位置的电偶极子在观测位置 $\vec{r}$ 处产生 的响应 (电场或磁场) 表示为 $f(p, \theta, \phi, \vec{l}, \vec{r})$, 其中 $f(\cdots)$ 可 以表示辐射电磁场 $E_{x} 、 E_{y} 、 E_{z} 、 B_{x} 、 B_{y} 、 B_{z}$ 的计算式, $\theta$ 和 $\phi$ 为已知参数, $p=I d l, p$ 表示天线 $l$ 位置处的电偶 极矩, $I$ 为天线中电流强度, $d l$ 表示 $l$ 位置的偶极子单位 长度, $\vec{l}$ 和 $\vec{r}$ 分别为电偶极天线和观测点的相对于坐标原 点的位置矢量。因此, 有限长度电性天线的电磁场计算式 如下[21]。 


$$
\int_{L} f(p, \theta, \phi, \vec{l}, \vec{r}) d l
$$

\subsection{2. 传统电性天线电磁场的积分方法}

传统电性天线辐射电磁场的积分计算方法主要采用 电偶极天线等效方法和均匀密集分割积分方法。对于电偶 极子天线等效方法, 是将有限长度电性天线看作电偶极天 线, 此时积分式(3)变为:

$$
\int_{L} f(p, \theta, \phi, \vec{l}, \vec{r}) d l \cong f\left(P, \theta, \phi, \overrightarrow{l_{p}}, \vec{r}\right)
$$

其中, $\overrightarrow{l_{P}}$ 表示有限长电性天线中心位置的位置矢量。 而均匀密集分割积分方法是将积分式(3)均匀离散化 为众多的电偶极天线响应之和的形式。

$$
\int_{L} f(p, \theta, \phi, \vec{l}, \vec{r}) d l \cong \sum_{i=1}^{N} f\left(p_{i}, \theta, \phi, \vec{l}_{i}, \vec{r}\right)
$$

其中 $N$ 为均匀分割的分点数, 一般为保证计算精度, 对于长度 100 米以上的天线, $N$ 取值不小 100 。

\section{3. 层状海洋电性天线电磁场的非均匀稀疏分割 计算方法}

为解决传统方法在计算效率和精度难以兼顾的问题, 本节将建立基于勒让德多项式零点和切比雪夫多项式零 点的非均匀稀疏分割计算方法, 以保证计算精度的同时极 大减少积分节点数，提升了计算效率。同时，本节将从计 算精度和效率两个角度对基于勒让德多项式零点和切比 雪夫多项式零点的两种非均匀稀疏分割计算方法性能就 行对比, 给出最优选择建议。

\section{1. 基于高斯勒让德积分的非均匀稀疏分割计算方法}

基于高斯勒让德积分的非均匀稀疏分割计算方法, 利 用具备正交性的勒让德多项式的零点作为高斯积分点, 采 用拉格朗日插值多项式计算积分系数, 并通过对积分核函 数的构造实现快速精确的计算。其计算方法流程如下。

第一步, 求 $n+1$ 次勒让德多项式 $P_{n+1}(x)$ 的 $n+1$ 个零 点 $x_{k}, k=0,1,2, \cdots, n$, 用于后续产生积分节点。 $P_{n+1}(x)$ 可以通过勒让德多项式的递推关系得到, 递推关系式为

$$
\left\{\begin{array}{l}
P_{0}(x)=1 \\
P_{1}(x)=x \\
P_{n}(x)=\frac{1}{2^{n}(n !)} \frac{d^{n}}{d x^{n}}\left(x^{2}-1\right)^{n}
\end{array}\right.
$$

第二步，计算各节点对应的拉格朗日插值基函数 $L_{k}(x)$, 用于计算各积分节点对应的积分系数。 $x_{k}$ 对应的 拉格朗日插值基函数 $L_{k}(x)$ 的计算式如下

$$
L_{k}(x)=\prod_{\substack{j=0, j \neq k}}^{n} \frac{\left(x-x_{j}\right)}{\left(x_{k}-x_{j}\right)}
$$

第三步, 计算各积分节点对应的积分系数 $A_{k}$ 。

$$
A_{k}=\int_{-1}^{1} L_{k}(x) d x, k=0,1, \cdots, n
$$

第四步, 进行积分区间变换, 调整积分区间与有限长 度电性天线长度范围匹配。

积分节点 $x_{k}$ 及其对应积分系数 $A_{k}$ 与积分区间无关, 仅与分点数 (积分阶数) 有关。因此, 算法实现时可以将 常用阶数的积分节点和积分系数存储以备随时调用, 以此 获得额外的加速计算效率。积分区间变换式如下:

$$
l_{k}=\frac{1}{2}\left[(b-a) x_{k}+a+b\right], x_{k} \in[-1,1]
$$

其中 $a$ 和 $b$ 分别为电性天线长度范围对应的坐标。

当计算天线的 $X$ 轴分量时, $b=L_{x} / 2, a=-L_{x} / 2$, 计算得到的为天线 $X$ 轴分量各分点坐标 $l_{k}^{x}$;

当计算天线的 $Y$ 轴分量时, $b=L_{y} / 2, a=-L_{y} / 2$, 计算得到的为天线 $Y$ 轴分量各分点坐标 $l_{k}^{y}$;

当计算天线的 $Z$ 轴分量时, $b=L_{z} / 2, a=-L_{z} / 2$, 计算得到的为天线的 $Z$ 轴分量各分点坐标 $l_{k}^{z}$ 。

求解过程中的 $x_{k}$ 保持不变。 $L_{x} 、 L_{y}$ 和 $L_{z}$ 通过投影 变化得到

$$
\left\{\begin{array}{l}
L_{x}=L \cos (\varphi) \cos (\theta) \\
L_{y}=L \cos (\varphi) \sin (\theta) \\
L_{z}=L \sin (\varphi)
\end{array}\right.
$$

第五步, 带入式(25)式计算任意姿态有限长度电性天 线的电磁场

$$
\int_{L} f\left(p_{i}, \theta, \varphi, \vec{l}_{i}, \vec{r}\right) d l_{i} \cong \frac{L}{2} \sum_{k=0}^{n} A_{k} f\left(p_{k}, \theta, \varphi, \overrightarrow{l_{k}}, \vec{r}\right)
$$

基于高斯-勒让德积分方法, 以 $\mathrm{n}+1$ 次勒让德多项式的 零点作为积分节点构建代数精度为 $2 n+1$ 次的积分求解方 法, 以保证非均匀稀疏分割积分方法的计算精度。

\section{2. 基于高斯切比雪夫积分的非均匀稀疏分割计算方法}

基于高斯切比雪夫积分的非均匀稀疏分割计算方法, 利用具备带权正交性的切比雪夫多项式的零点作为高斯 积分点, 采用带权值的拉格朗日插值多项式计算积分系数, 
并通过对积分核函数的构造实现快速精确的计算。其计算 方法流程如下。

第一步, 求 $n+1$ 次切比雪夫多项式的 $n+1$ 个零点, 用于 后续产生积分节点。切比雪夫多项式满足下面的递推关系 式

$$
\left\{\begin{array}{l}
T_{n+1}(x)=2 x T_{n}(x)-T_{n-1}(x) \\
T_{2}(x)=2 x^{2}-1 \\
T_{1}(x)=x \\
T_{0}(x)=1
\end{array}, \mathrm{n}=1,2,3, \cdots\right.
$$

其对应的 $n+1$ 个零点为:

$$
x_{k}=\cos \left(\frac{2 k+1}{2 n+2} \pi\right), k=0,1,2, \cdots, n
$$

第二步，计算各节点的拉格朗日插值基函数 $l_{k}(x)$, 用于计算各积分节点对应的积分系数。 $x_{k}$ 对应的拉格朗 日插值基函数 $l_{k}(x)$ 为:

$$
l_{k}(x)=\prod_{\substack{j=0, j \neq k}}^{n} \frac{\left(x-x_{j}\right)}{\left(x_{k}-x_{j}\right)}
$$

第三步, 求各积分节点带权值 $\rho(x)=\frac{1}{\sqrt{1-x^{2}}}$ 的积分 系数 $A_{k}$, 其中 $x \in[-1,1]$ 。积分系数计算式为:

$$
A_{k}=\int_{-1}^{1} \frac{l_{k}(x)}{\sqrt{1-x^{2}}} d x=\frac{\pi}{n+1}, k=0,1,2, \cdots \mathrm{n}
$$

第四步, 积分区间变换, 调整积分区间与有限长度电 性天线长度范围匹配。方法同3.2节第四步。

第五步, 带入(30)式中计算任意姿态有限长电性天线 的电磁场。

$$
\begin{aligned}
& \int_{L} f(p, \theta, \phi, \vec{l}, \vec{r}) d l \\
& \cong \frac{L}{2} \frac{\pi}{n+1} \sum_{k=0}^{n} f\left(p_{k}, \theta, \phi, \overrightarrow{l_{k}}, \overrightarrow{r_{k}}\right) \sqrt{1-x_{k}^{2}}
\end{aligned}
$$

基于高斯-切比雪夫积分方法进行层状海洋任意姿态 有限长电性天线电磁场的快速计算方法, 是以 $\mathrm{n}+1$ 次切比 雪夫多项式的零点作为积分节点构建代数精度为 $2 n+1$ 次 的积分求解方法, 以保证非均匀稀疏分割积分方法的计算 精度。

\begin{tabular}{|c|c|c|c|}
\hline \multirow[b]{2}{*}{ 参数符号 } & \multicolumn{3}{|l|}{ 参数信息 } \\
\hline & 参数名称 & $\begin{array}{l}\text { 计量单 } \\
\text { 位 }\end{array}$ & 取值 \\
\hline I & 天线电流 & $\mathrm{A}$ & 1 \\
\hline $\mathrm{L}$ & 电线长度 & $\mathrm{m}$ & 300 \\
\hline$\theta$ & $\begin{array}{l}\text { 水平面内天线 } \\
\text { 与X轴的夹角 }\end{array}$ & $\mathrm{rad}$ & $\pi / 3$ \\
\hline$\varphi$ & $\begin{array}{l}\text { 天线与水平面 } \\
\text { 的夹角 }\end{array}$ & $\mathrm{rad}$ & $\pi / 6$ \\
\hline $\mathrm{f}$ & 工作频率 & $\mathrm{Hz}$ & 0.25 \\
\hline $\mathrm{t}$ & 时域观测时间 & $\mathrm{s}$ & $\begin{array}{l}0.01 \mathrm{~s}-100 \mathrm{~s} \text {, 对数等间隔取 } \\
\text { 样 }\end{array}$ \\
\hline $\mathrm{H}$ & $\begin{array}{l}\text { 天线距离水面 } \\
\text { 距离 }\end{array}$ & $\mathrm{m}$ & 120 \\
\hline $\mathrm{d}_{1}$ & 海水深度 & $\mathrm{m}$ & 220 \\
\hline$\mu_{0}$ & 真空磁导率 & $\mathrm{H} / \mathrm{m}$ & 各层介质默认 $\mu_{0}$ \\
\hline$\varepsilon_{0}$ & 真空介电常数 & $\mathrm{F} / \mathrm{m}$ & 各层介质默认 $\varepsilon_{0}$ \\
\hline$\sigma_{\mathrm{i}}$ & 各层电导率 & $\mathrm{S} / \mathrm{m}$ & $\begin{array}{l}\text { 海水电导率为 } 0.33 \text {; 海底电 } \\
\text { 导率为 } 2\end{array}$ \\
\hline $\begin{array}{l}\text { n-gauleg, } \\
\text { n-gauche }\end{array}$ & 积分节点数 & - & $\begin{array}{l}\text { 通常取值 } 7 \sim 13 \text {, 这里取值 } \\
12 \text {, 均匀精细分割点数 } 200\end{array}$ \\
\hline tol & 误差限 & - & $\begin{array}{l}\text { 通常取值1e-8 1e-12, 这里 } \\
\text { 取值为1e-12 }\end{array}$ \\
\hline
\end{tabular}

\section{3. 模拟计算结果与性能分析}

为验证本论文提出的方法, 对比两种非均匀稀疏分割 计算方法的效果和性能, 表 1 给出了模拟仿真计算对比案 例中的参数。其中海底设置为均匀半空间。
表1 模拟仿真参数列表。

图7-图14分别给出了海底沿 $\mathrm{x}$ 轴和 $\mathrm{y}$ 轴方向 $0-1 \mathrm{~km}$ 范围 内磁感应强度总场及各分量磁感应强、电场总场和各分量 电场的幅度和相位分布曲线。从响应幅度曲线的计算结果 可知, (1). 电偶极天线近似计算结果误差最大, 甚至短偏 移距时的分布曲线形态已无法反应真实分布曲线形态, 如 图8和图12给出的电场总场及各分量幅度分布曲线; (2). 两种非均匀稀疏分割计算方法几乎完全重合, 两种方法的 计算精度一致。从相位分布曲线计算结果可知, (1). 电偶 极天线近似计算结果的相位分布在短偏移距时存在较大 误差, 如图10和图 14; (2).两种非均匀稀疏分割计算方法 的相位曲线几乎完全重合。综合幅度和相位曲线结果可知, 两种非均匀稀疏分割计算结果与均匀密集分割计算结果 精度相当, 幅度和相位分布曲线具有很好的一致性。
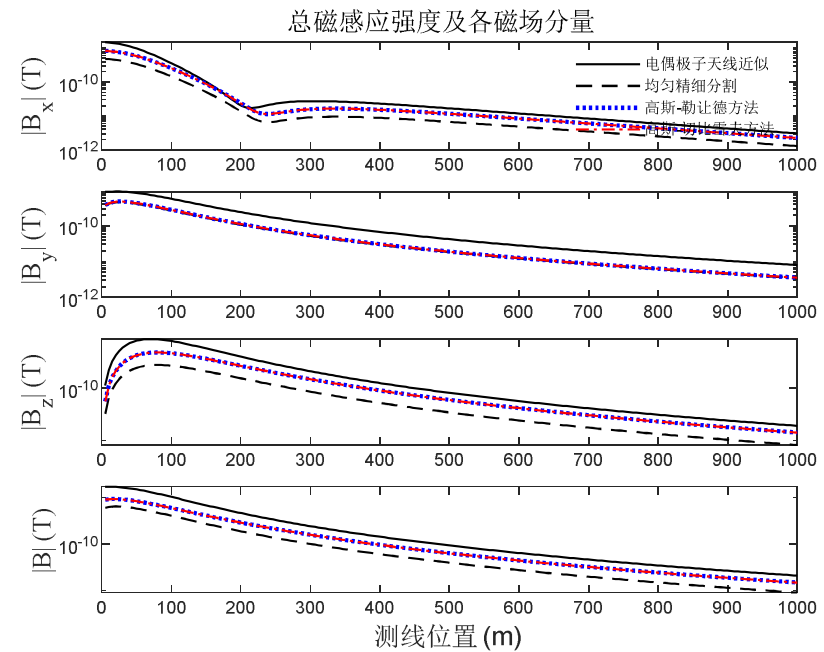

图7 海底x方向 $0-1 \mathrm{~km}$ 范围内磁感应总场及各分量的幅度分布曲线。 


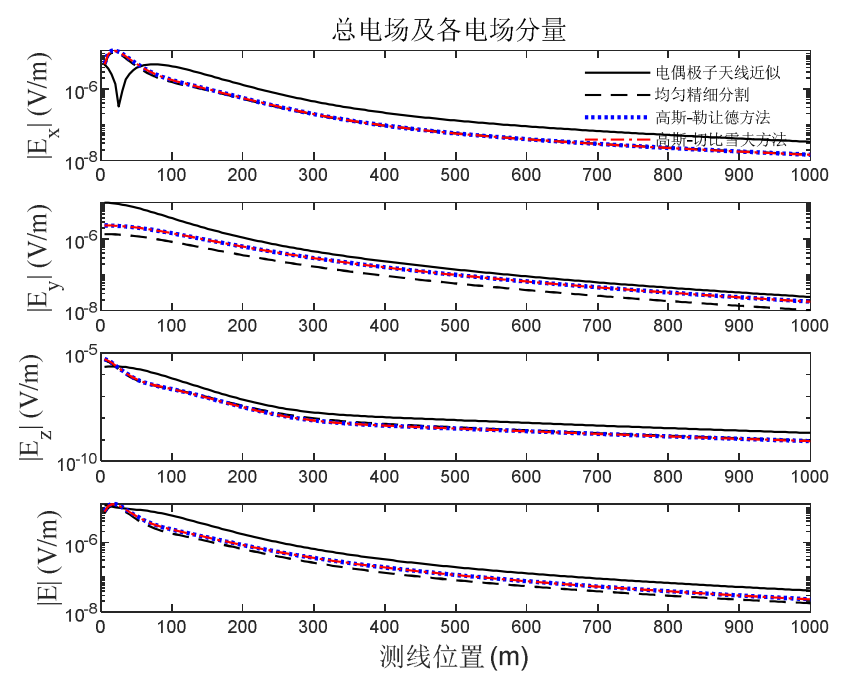

图8 海底 $x$ 方向0-1 km范围内电场总场及各分量的幅度分布曲线。
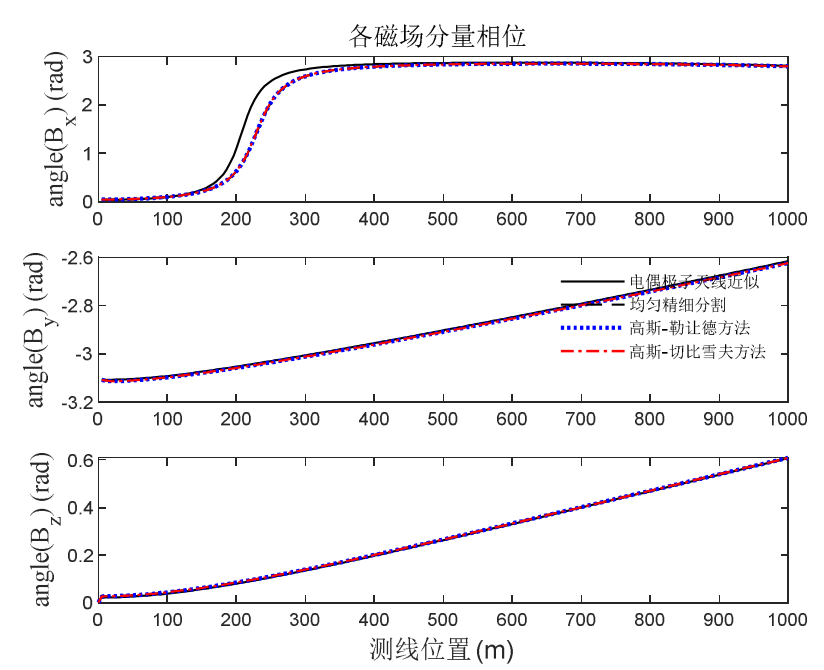

图9 海底x方向0-1 km范围内各磁感应强度分量的相位分布曲线。


图10 海底x方向 $0-1 \mathrm{~km}$ 范围内电场各分量的相位分布曲线。

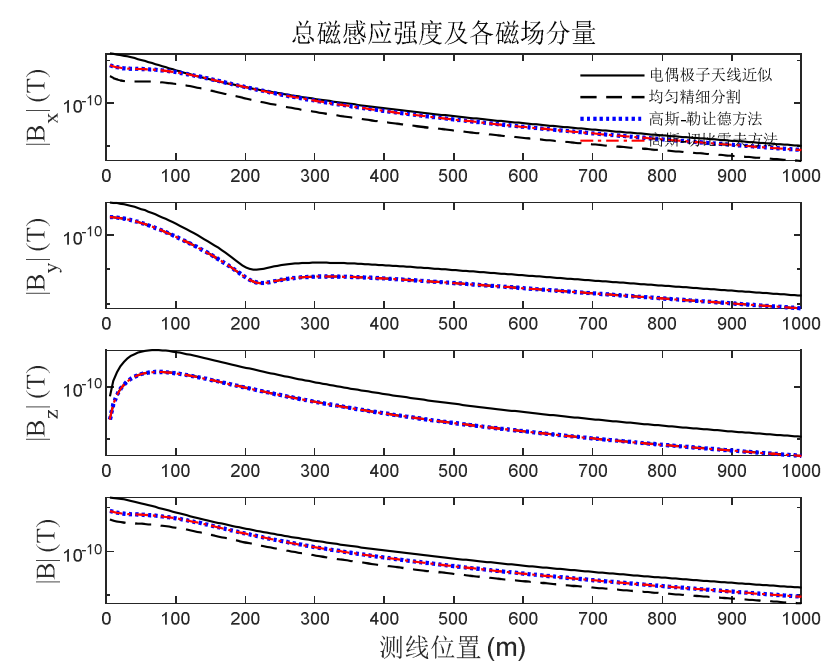

图11 海底y方向0-1 km范围内磁感应总场及各分量的幅度分布曲线。

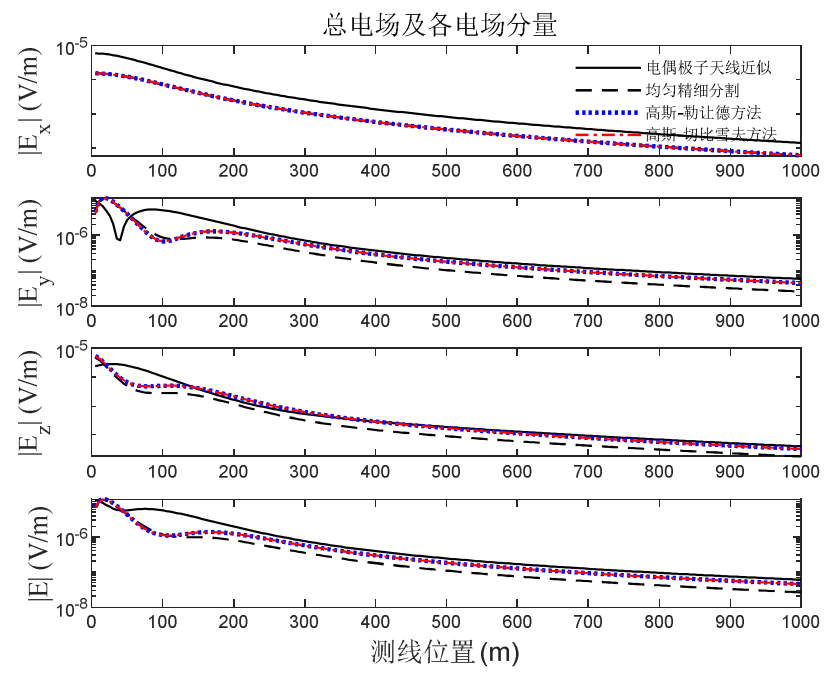

图12 海底y方向 $0-1 \mathrm{~km}$ 范围内电场总场及各分量的幅度分布曲线。
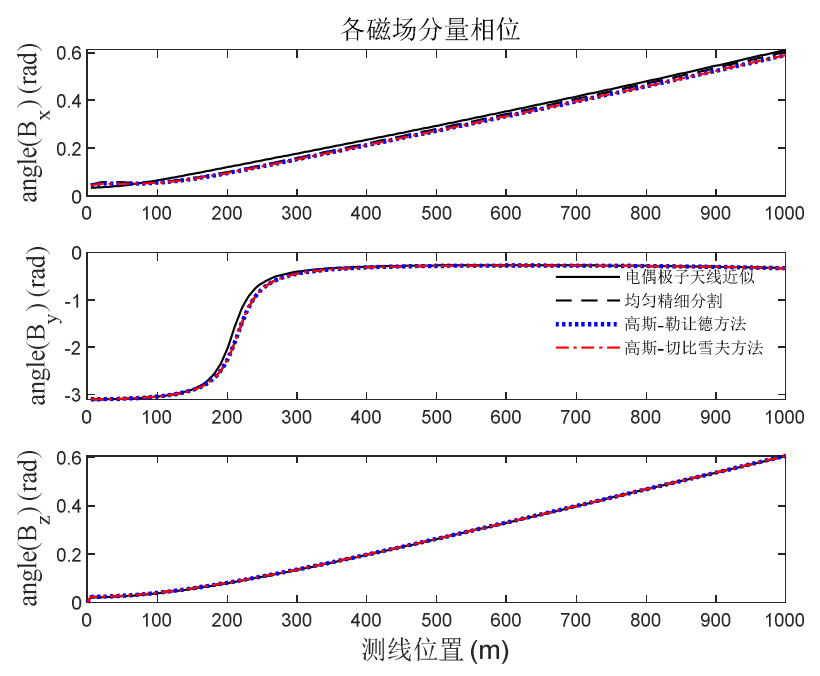

图13 海底y方向 $0-1 \mathrm{~km}$ 范围内各磁感应强度分量的相位分布曲线。 

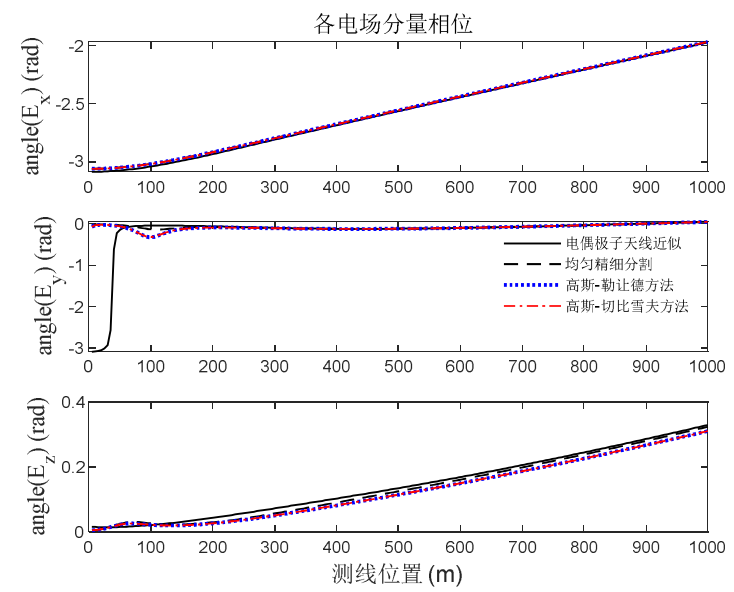

图14 海底y方向0-1 km范围内电场各分量的相位分布曲线。

图15和图 16 出了海底 $(0,500 \mathrm{~m})$ 位置的磁感应总场、 各磁场分量、电场总场和各电场分量的负阶跃响应曲线。 仿真结果表明, (1). 电偶极天线近似方法计算误差最大, 如图16中总电场的响应曲线; (2).两种非均匀稀疏分割方 法计算结果有高度的一致性, 且与均匀精细分割方法计算 结果基本重合。结果表明两种非均匀稀疏分割方法计算结 果精度与均匀精细分割方法的计算结果相当。
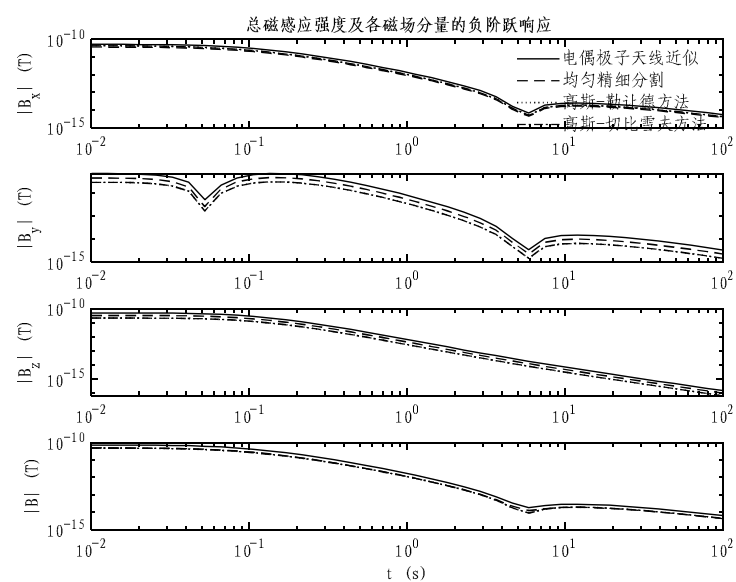

图15 海底 $(0,500 \mathrm{~m})$ 位置的磁感应总场及各分量的负阶跃响应。
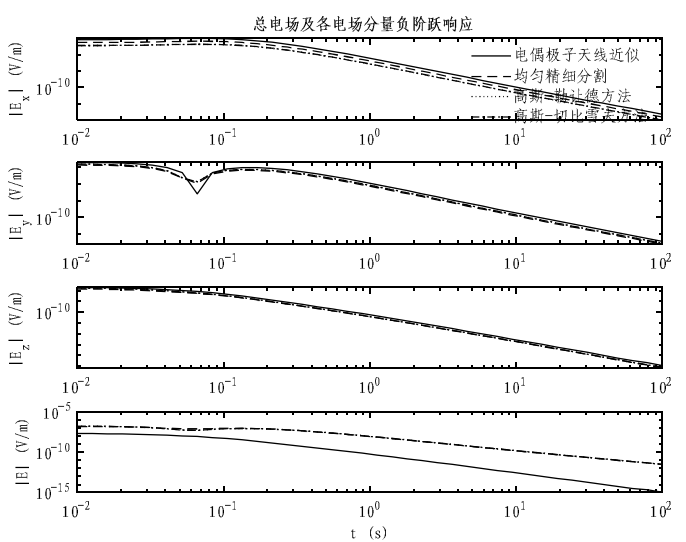

图16 海底 $(0,500 \mathrm{~m})$ 位置的电场总场及各分量的负阶跃响应。
为对比分析两种非均匀稀疏分割计算方法的计算效 率性能, 采用相同的计算平台和仿真软件进行仿真计算分 析。其中, 计算机配置为Win7系统, 六核Intel i5-8400, 主频2.80GHz, 8GB RAM, 仿真软件采用MatlabR2018a-64 位版本。频域仿真计算性能对比单频点计算，200个观测 点六个分量的仿真计算总耗时; 时域仿真计算性能对比从 $0.01 \mathrm{~s}-100 \mathrm{~s}$ 时间段对数等间隔地取 40 个计算时刻的仿真计 算总耗时。表2给出了两种方法的计算耗时。推荐使用的 非均匀稀疏分割计算参数为积分节点数 7-13, 误差限 1e-8-1e-12。

表1 计算耗时统计, 单位 $\mathrm{S}$ 。

\begin{tabular}{lll}
\hline 方法 & 频域 & 时域 \\
\hline 200点均匀精细分割 & 304.559180 & 76.599621 \\
12点高斯-勒让德方法 & 20.211051 & 72.247585 \\
12点高斯-切比雪夫方法 & 20.849726 & 7.361508 \\
\hline
\end{tabular}

注:计算平台配置为Win7系统, 六核Intel i5-8400, 主频 $2.80 \mathrm{GHz}, 8 \mathrm{~GB}$ RAM，仿真软件为MatlabR2018a-64位版本。

对比两种非均匀稀疏分割计算方法与均匀精细分割 计算方法的总耗时, 结果表明: (1).非均匀稀疏分割计算 方法对于频域仿真计算能够获得极大的性能提升, 性能提 升近15倍; (2).对于时域仿真计算, 基于高斯-勒让德积分 的非均匀稀疏分割计算方法带来的性能提升有效，而基于 高斯-切比雪夫积分的非均匀稀疏分割方法能带来约 10 倍 以上的性能提升。

综合对比两种非均匀稀疏分割计算方法、电偶极天线 近似计算方法和均匀密集分割计算方法的计算结果精度 和计算效率可知, 在保证计算精度的情况下, 基于高斯切比雪夫积分的非均匀稀疏分割计算方法效果最佳, 其对 层状海洋有限长度电性天线辐射电磁场的时频域计算效 率的提升达 10 倍以上; 其次是基于高斯-勒让德积分的方 法, 其对频域仿真计算效率的提升与前者方法相当。

\section{4. 结论}

本论文针对当前层状海洋电性天线电磁场时频域计算 精度和效率难以兼顾的问题, 引入了两种基于高斯积分节 点 (勒让德多项式零点和切比雪夫多项式零点) 的非均匀 稀疏分割数值积分计算方法, 构建了相应的层状海洋电性 天线电磁场的快速计算方法, 通过从计算精度和计算效率 两个角度与传统计算方法进行对比分析, 证明了在保证计 算精度的前提下，两种非均匀稀疏分割计算方法能够实现 有限长度电性天线电磁场的快速计算, 极大地提升了计算 效率, 其中基于高斯-切比雪夫积分的非均匀稀疏分割积分 方法对有限长度电性天线电磁场的时频域计算效果最佳, 能够实现工程应用中数据解释的效率和准确性的提升。

\section{参考文献}

[1] 何展翔, 余刚. 海洋电磁勘探技术及新进展 $[\mathrm{J}]$. 勘探地球物理 进展,2008，31(1):2-10。 
[2] NIGEL EDWARDS. MARINE CONTROLLED SOURCE ELECTROMAGNETICS: PRINCIPLES, METHODOLOGIES, FUTURE COMMERCIAL APPLICATIONS [J]. Surveys in Geophysics, 2005, 26: 675-700.

[3] 朱忠民.海洋电磁响应有效信号增强与干扰压制方法研究 [D].北京：中国石油大学，2016。

[4] 周继瑜.海洋电磁信号空气波压制技术研究[D].吉林：吉林 大学, 2014。

[5] Rong Liu, Jianin Liu, Jianxin Wang, Zhuo Liu, Rongwen Guo. 1D EM response modeling with arbitrary source-receiver geometry based on vector potential and its implementation in Matlab [J]. Geophysics, 2020. 85 (3): F27-F38. DOI: 10.1190/geo2019-0224.1.

[6] Ping $\mathrm{Yu}$ et al. The analysis on Marine Controlled-Source Electromagnetic Research Status [J]. IOP Conf. Ser.:Earth and Environmental Science, 2019. 252 (5).

[7] 翟景红.海洋聚焦电磁法理论与实验研究[D].2018, 北京: 中 国地质大学(北京), 2018: 13-33。

[8] Key K. 1D inversion of multicomponent, multifrequency marine CSEM data: Methodology and synthetic studies for resolving thin resistive layers [J]. Geophysics, 2009, 74 (2).

[9] 兰怀慷. 全参数海洋可控源电磁法一维反演[D].2018, 桂林: 桂林理工大学, 2018:9-31。

[10] 刘颖、李予国,层状各向异性介质中任意取向电偶源的海洋 电磁响应.石油地球物理勘探,2015.50(4): p.755-765。

[11] Nabighian M N. Electromagnetic Methods in Applied Geophysics: Volume 1, Theory [M]. Society of Exploration Geophysicists, 1991: 46-48.
[12] Kaufman A A, Keller G V. Frequency and transient soundings [M]. Springer, 1983.

[13] 渠晓东,甚低频/极低频电磁法在目标探测中的应用 [D]. 北 京: 中国科学院大学, 2017。

[14] 时宗洋,半航空瞬变电磁系统探测方法研究[D].中国科学院 大学:北京, 2018。

[15] 张建国.可控源电磁勘探方法与技术研究[D].北京: 中国科 学院大学,2014。

[16] 卢新城, 龚沈光, 陈新刚, 水平 $\mathrm{n}$ 层导电介质中时谐垂直电 偶极子的电磁场. 地球物理学进展, 2004。

[17] KerryKey, OCCAM1DCSEM An Open-Source Inversion Program for Generating Smooth 1D Models from Controlled-Source Electromagnetic and Magnetotelluric Data [R]. 2011, Scripps Institution of Oceanography, University of California, San Diego.

[18] Andrew Pethick, Planning and 4D Visualisation of the marine controlled source electromagnetic method [D]. 2008, Department of Exploration Geophysics, Curtin University of Technology.

[19] 朴化荣. 电磁测深法原理[M]. 北京, 地质出版社, 1990。

[20] 张博.基于非结构有限元的频率/时间域航空电磁系统仿真 研究[D].长春: 吉林大学, 2017:35-37。

[21] Shi Z, Liu L, Xiao P, et al. Simulation and Analysis of the effect of ungrounded rectangular loop distributed parameters on TEM response [J]. Journal of Applied Geophysics, 2018, 149. 